\title{
Práticas assistenciais em partos de risco habitual assistidos por enfermeiras obstétricas
}

Care practices in normal-risk births assisted by obstetric nurses

Prácticas asistenciales en partos de riesgo normal asistidos por enfermeras obstétricas

Simone Konzen Ritter ${ }^{1}$ iD https://orcid.org/0000-0002-7623-6461

Annelise de Carvalho Gonçalves ${ }^{1}$ id https://orcid.org/0000-0002-8770-0653

Helga Geremias Gouveia ${ }^{1}$ id https://orcid.org/0000-0003-4980-4141

\section{Como citar:}

Ritter SK, Gonçalves AC, Gouveia HG. Práticas assistenciais em partos de risco habitual assistidos por enfermeiras obstétricas. Acta Paul Enferm. 2020;33:APE20180284.

http://dx.doi.org/10.37689/ acta-ape/2020A00284

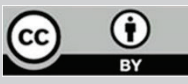

Descritores

Enfermagem obstétrica; Enfermeiras obstétricas; Parto humanizado; Trabalho de parto

Keywords

Obstetric nursing; Nurse midwives;Humanizing delivery; Labor, obstetric

Descriptores

Enfermería obstétrica; Enfermeras obstetrices; Parto humanizado; Trabajo de parto

Submetido 3 de Dezembro de 2018

Aceito

30 de Setembro de 2019

\section{Autor correspondente}

Simone Konzen Ritter

E-mail: simone.ritter@ufrgs.br

\section{Resumo}

Objetivo: Comparar as práticas assistenciais em partos de risco habitual assistidos por enfermeiras obstétricas em um hospital público de Porto Alegre/RS no ano de 2013 - início do modelo colaborativo na instituição com as práticas assistenciais realizadas no ano de 2016.

Métodos: Estudo transversal, retrospectivo, analítico, realizado no centro obstétrico de um hospital público de Porto Alegre/RS, com 186 parturientes de risco habitual com parto assistido por enfermeiras obstétricas no período de 2013 e 2016. Constituíram critérios de inclusão gestantes de risco habitual, durante o prénatal e admissão hospitalar, com feto único, recém-nascido vivo, a termo (idade gestacional de 37 a 41 semanas) e em apresentação cefálica fletida. Foram excluídas parturientes que ingressaram na instituição em período expulsivo e as com informações incompletas em prontuário. Os dados provenientes do estudo foram agrupados sob a forma de banco de dados e analisados no Statistical Package for the Social Sciences (SPSS) versão 25.0. Para análise estatística foi utilizado o Teste Qui-quadrado de Pearson e 0 Teste Exato de Fischer, para comparar proporções.

Resultados: A comparação das práticas assistenciais nos anos estudados revelou redução de intervenções como tricotomia $(-100,0 \%)$, uso de supositório retal $(-85,8 \%)$, posição litotômica $(-85,0 \%)$, uso de medicamentos para alívio da dor $(-79,0 \%)$, analgesia epidural $(-79,0 \%)$, uso de ocitocina $(-73,3 \%)$, cateterização venosa $(-60,5 \%)$, cardiotocografia $(-51,1 \%)$, tonsura $(-38,5 \%)$, bola obstétrica $(-31,0 \%)$ e posição semissentada $(-5,4 \%)$; e aumento percentual de práticas como a mudança de posição $(+828,6 \%)$, rebozo (+167,3\%), posição de cócoras (+100,0\%), posição quatro apoios (+100,0\%), posição lateral direita $(+100,0 \%)$, posição lateral esquerda $(+100,0 \%)$, uso de partograma $(+43,3 \%)$, massagem terapêutica $(+33,4 \%)$, clampeamento tardio do cordão umbilical $(+37,3 \%)$, contato pele a pele $(+33,2 \%)$, amniotomia $(+16,7 \%)$ e dieta líquida $(+11,5 \%)$.

Conclusão: Frente ao modelo predominante de assistência obstétrica no Brasil, centrado no médico obstetra e em práticas intervencionistas, o modelo colaborativo de assistência ao parto, com atuação das enfermeiras obstétricas, mostra-se como um caminho para a atenção às mulheres, com respeito à fisiologia do parto e ao protagonismo da mulher.

\section{Abstract}

Objective: To compare the care practices in normal-risk births assisted by obstetric nurses in a public hospital in Porto Alegre, Brazil in 2013, when the collaborative model was first implemented in that institute, with care practices employed in 2016. 
Methods: A cross-sectional, retrospective, analytical study conducted in the obstetric center of a public hospital in Porto Alegre, Brazil with 186 women at normal-risk labor assisted by obstetric nurses in 2013-2016. Inclusion criteria were pregnant women at normal risk during prenatal care and hospital admission, with a single fetus born alive at full term (gestational age, 37-41 weeks) with a well-flexed cephalic presentation. Parturients admitted to the institute during the expulsion phase and those with incomplete information in their medical records were excluded. Data from the study were grouped into a database and analyzed using Statistical Package for the Social Sciences software, version 25.0. Pearson's chi-square test and Fisher's exact test were used to compare proportions.

Results: The comparison of care practices revealed a reduction in interventions such as trichotomy $(-100.0 \%)$, rectal suppository use (-85.8\%), lithotomic position $(-85.0 \%)$, use of pain-relief medication $(-79.0 \%)$, epidural analgesia $(-79.0 \%)$, oxytocin use $(-73.3 \%)$, venous catheterization $(-60.5 \%)$, cardiotocography $(-51.1 \%)$, pubic-hair trimming $(-38.5 \%)$, birth ball $(-31.0 \%)$, semi-sitting position $(-5.4 \%)$, and an increase in practices such as change in position (+828.6\%), rebozo (+167.3\%), squatting position (+100.0\%), all-fours position (+100.0\%), right lateral position (+100.0\%), left lateral position $(+100.0 \%)$, use of partograms (+43.3\%), therapeutic massage (+33.4\%), late umbilical-cord clamping (+37.3\%), skin-to-skin contact (+33.2\%), amniotomy (+16.7\%), and liquid diet (+11.5\%).

Conclusion: In the context of the predominant model of obstetric care in Brazil, centered on the obstetric physician and interventionist practices, the collaborative model of childbirth care with the active participation of obstetric nurses is a good way to take care of women giving birth, respecting the physiology of childbirth, and the woman's protagonism.

\section{Resumen}

Objetivo: Comparar las prácticas asistenciales en partos de riesgo normal asistidos por enfermeras obstétricas en un hospital público de Porto Alegre/RS en el año 2013 —inicio del modelo colaborativo en la institución— con las prácticas asistenciales realizadas en el año 2016.

Métodos: Estudio transversal, retrospectivo, analítico, realizado en el centro obstétrico de un hospital público de Porto Alegre/RS, con 186 parturientas de riesgo normal con parto asistido por enfermeras obstétricas en el período de 2013 y 2016. Los criterios de inclusión fueron embarazadas de riesgo normal, durante la atención prenatal y admisión hospitalaria, con feto único, recién nacido vivo, a término (edad gestacional entre 37 y 41 semanas) y presentación cefálica flexionada. Se excluyeron parturientas que ingresaron a la institución en período expulsivo y las que tenían información incompleta en la historia clínica. Los datos provenientes del estudio se agruparon bajo la forma de banco de datos y se analizaron en el Statistical Package for the Social Sciences (SPSS) versión 25.0. Para el análisis estadístico se utilizó la prueba $\chi^{2}$ de Pearson y la Prueba exacta de Fisher para comparar proporciones.

Resultados: La comparación de las prácticas asistenciales durante los años estudiados reveló una reducción de intervenciones como tricotomía (-100,0\%), uso de supositorio rectal (-85,8\%), posición de litotomía (-85,0\%), uso de medicamentos para aliviar el dolor (-79,0\%), analgesia epidural (-79,0\%), uso de oxitocina $(-73,3 \%)$, cateterización venosa $(-60,5 \%)$, cardiotocografía $(-51,1 \%)$, tonsura $(-38,5 \%)$, pelota obstétrica $(-31,0 \%)$ y posición semisentada $(-5,4 \%)$; y un aumento en el porcentaje de prácticas como el cambio de posición (+828,6\%), rebozo (+167,3\%), posición de cuclillas (+100,0\%), posición cuatro apoyos $(+100,0 \%)$, posición de lado derecho $(+100,0 \%)$, posición de lado izquierdo $(+100,0 \%)$, uso de partograma $(+43,3 \%)$, masajes terapéuticos (+33,4\%), pinzamiento tardío del cordón umbilical (+37,3\%), contacto piel con piel (+33,2\%), amniotomía (+16,7\%) y dieta líquida (+11,5\%).

Conclusión: Frente al modelo predominante de atención obstétrica en Brasil, centrado en el médico obstetra y en prácticas intervencionistas, el modelo colaborativo de atención al parto, con actuación de enfermeras obstétricas, demuestra ser un camino para la atención a las mujeres, respecto a la fisiología del parto y al protagonismo de la mujer.

\section{Introdução}

O modelo predominante e tradicional de assistência obstétrica no Brasil é centrado no médico obstetra e na atenção hospitalar. O Ministério da Saúde (MS) incentiva a incorporação da enfermeira obstétrica nas equipes hospitalares e aposta na sua contribuição para redução no uso das intervençôes e das cesáreas desnecessárias. ${ }^{(1)}$

No Brasil, a participação de enfermeiras obstétricas no parto vaginal é limitada. Em estudo realizado em maternidades brasileiras, 16,2\% dos partos vaginais foram assistidos por enfermeiras obstétricas, nos quais as boas práticas foram significativamente mais frequentes. ${ }^{(2)}$

A assistência ao parto e nascimento de baixo risco pode ser realizada tanto por médico obstetra quanto por enfermeira obstétrica e obstetriz. ${ }^{(3)}$ É recomendado que os gestores proporcionem condiçóes para a implementação do modelo colaborativo de assistência, por apresentar vantagens em relação à redução de intervençóes e maior satisfação das mulheres. ${ }^{(3)}$

A assistência obstétrica no modelo colaborativo significa a integração do médico e da enfermeira obstétrica na equipe. A enfermeira obstétrica assume a assistência das mulheres de risco habitual, assegurada a possibilidade de referência imediata ao médico obstetra em casos de complicações. ${ }^{(4)}$

Este estudo objetiva comparar as práticas assistenciais em partos de risco habitual assistidos por enfermeiras obstétricas em um hospital público de Porto Alegre/RS no ano de 2013 - início do modelo colaborativo na instituição - com as práticas assistenciais realizadas no ano de 2016. 
A partir do exposto, este estudo apresenta como questão de pesquisa: "Houve aumento da realização de boas práticas de atenção em partos assistidos por enfermeiras obstétricas?", com o intuito de contribuir para a produção de evidências científicas acerca da assistência ao parto e nascimento no modelo colaborativo no Brasil.

\section{Métodos}

Estudo transversal, retrospectivo, analítico, realizado no Centro Obstétrico do Hospital Nossa Senhora da Conceição (HNSC), localizado no município de Porto Alegre/RS, Brasil.

A amostra foi constituída por 186 parturientes de risco habitual com parto assistido por enfermeiras obstétricas nos anos de 2013 e 2016. Neste período as enfermeiras obstétricas da instituição assistiram 621 partos, com percentuais que variaram entre $4,9 \%$ (138 partos) em 2013 e 22,2\% (483 partos) em 2016. Foram incluídas na amostra 30\% das parturientes com parto assistido por enfermeiras obstétricas por ano, por ser este o percentual atingido no ano de realização deste estudo (2017), representando, assim, 41 parturientes em 2013 e 145 em 2016. A amostra foi calculada com nível de confiança de $95 \%$ e margem de erro de cinco pontos percentuais e selecionada a partir de amostragem aleatória simples.

Constituíram critérios de inclusão gestantes de risco habitual, durante o pré-natal e admissão hospitalar, com feto único, recém-nascido vivo, a termo (idade gestacional de 37 a 41 semanas) e em apresentação cefálica fletida. Foram excluídas parturientes que ingressaram na instituição em período expulsivo e as com informaçóes incompletas em prontuário.

A coleta de dados ocorreu no período de março a agosto de 2017, a partir dos registros em prontuário da mulher. Os dados provenientes do estudo foram agrupados sob a forma de banco de dados e analisados no Statistical Package for the Social Sciences (SPSS) versão 25.0.
Para análise estatística foi utilizado o Teste Quiquadrado de Pearson e o Teste Exato de Fischer, para comparar proporçóes. Estabeleceu-se o nível de significância de $5 \%(\mathrm{p}<0,05)$. Foi realizada a variação percentual de todas as práticas assistenciais em estudo, para fins de comparação entre os anos de 2013 e 2016, a partir da seguinte fórmula:

Variação Percentual $=\frac{\text { Valor final }- \text { Valor inicial }}{\text { Valor inicial }} \times 100$

O projeto foi aprovado pelo Comitê de Ética em Pesquisa do Grupo Hospitalar Conceição, sob no 16278. Foram cumpridas as resoluçóes preconizadas pela legislação brasileira para pesquisas com seres humanos.

\section{Resultados}

Entre as 186 parturientes, a média de idade foi de $25,1 \pm 6,1$ anos (14-41 anos). A maioria era da cor branca $(68,8 \%)$, com ensino fundamental $(59,7 \%)$ e ocupação do lar (49,5\%).

Em relação à história obstétrica constatouse que $58,6 \%$ eram multíparas e $13,4 \%$ tiveram uma cesárea prévia. A média da idade gestacional foi de 39 semanas $\pm 1,2$ semanas $(37-41$ semanas) e 7,6 $\pm 2,5$ consultas de pré-natal $(2-14$ consultas), realizadas na rede pública em $92,5 \%$ das gestaçóes.

Em relação ao motivo de internação, 66,1\% ingressaram na instituição em trabalho de parto (TP) com bolsa íntegra, $17,7 \%$ em TP com bolsa rota, $9,7 \%$ com bolsa rota e $6,5 \%$ internaram por pósdatismo para indução do TP.

Acerca da caracterização dos recém-nascidos, 99,5\% apresentaram Índice de Apgar $\geq 7$ no $5^{\circ}$ minuto de vida. A média de peso do nascimento foi $3.233,2 \pm 407,3$ gramas $(2060-4250$ gramas) e $5,4 \%$ internaram em unidade neonatal por inadequada adaptação neonatal ou presença de infecçóes, como sepse neonatal e sífilis congênita.

A seguir, na tabela 1, estão apresentadas a comparação das práticas assistenciais no TP e parto nos anos de 2013 e 2016. 
Tabela 1. Comparação das práticas assistenciais no trabalho de parto e parto em partos assistidos por enfermeiras obstétricas

\begin{tabular}{|c|c|c|c|c|}
\hline Variável & $2013(\%)$ & $2016(\%)$ & VP (\%) & $p$-value \\
\hline Mudança de posição no TP & 4,9 & 45,5 & $+828,6$ & $0,00 \dagger$ \\
\hline Rebozo & 9,8 & 26,2 & $+167,3$ & $0,04 \dagger$ \\
\hline Posição de cócoras & 0,0 & 10,3 & $+100,0$ & $0,00 £$ \\
\hline Posição de quatro apoios & 0,0 & 6,2 & $+100,0$ & $0,00 £$ \\
\hline Posição lateral direita & 0,0 & 3,4 & $+100,0$ & $0,00 £$ \\
\hline $\begin{array}{l}\text { Posição lateral esquerda } \\
\text { Uso do partograma }\end{array}$ & $\begin{array}{c}0,0 \\
68,3\end{array}$ & $\begin{array}{l}14,5 \\
97,9\end{array}$ & $\begin{array}{l}+100,0 \\
+43,3\end{array}$ & $\begin{array}{l}0,00 £ \\
0,00 \dagger\end{array}$ \\
\hline Clampeamento tardio do cordão umbilical ${ }^{*}$ & 68,3 & 93,8 & $+37,3$ & $0,00 \dagger$ \\
\hline Massagem terapêutica & 61,0 & 81,4 & $+33,4$ & $0,01 \dagger$ \\
\hline Contato pele a pele $e^{\star \star}$ & 70,4 & 93,8 & $+33,2$ & $0,00 \dagger$ \\
\hline Amniotomia & 2,4 & 2,8 & $+16,7$ & $0,03+$ \\
\hline Dieta líquida por via oral & 87,8 & 97,9 & $+11,5$ & $0,01 £$ \\
\hline Presença de acompanhante & 95,1 & 99,3 & $+4,4$ & $0,12 £$ \\
\hline Banho de aspersão & 100,0 & 100,0 & 0,0 & $1,00 \dagger$ \\
\hline $\begin{array}{l}\text { Tricotomia } \\
\text { Supositório retal }\end{array}$ & $\begin{array}{l}7,3 \\
53,7\end{array}$ & $\begin{array}{l}0,0 \\
7,6\end{array}$ & $\begin{array}{l}-100,0 \\
-85,8\end{array}$ & $\begin{array}{l}0,01 £ \\
0,00 \dagger\end{array}$ \\
\hline Posição litotômica & 36,6 & 5,5 & $-85,0$ & $0,00 £$ \\
\hline $\begin{array}{l}\text { Medicamentos } \mathrm{p} / \text { alívio da dor } \\
\text { Analgesia epidural p/alívio da dor } \\
\text { Uso de ocitocina }\end{array}$ & $\begin{array}{l}29,3 \\
19,5 \\
43,9\end{array}$ & $\begin{array}{l}6,2 \\
4,1 \\
11,7\end{array}$ & $\begin{array}{l}-79,0 \\
-79,0 \\
-73,3\end{array}$ & $\begin{array}{l}0,00 £ \\
0,00 £ \\
0,00 \dagger\end{array}$ \\
\hline $\begin{array}{l}\text { Cateterização vesical } \\
\text { Episiotomia } \\
\text { Cateterização venosa }\end{array}$ & $\begin{array}{l}4,9 \\
4,9 \\
61,0\end{array}$ & $\begin{array}{l}1,4 \\
1,4 \\
24,1\end{array}$ & $\begin{array}{l}-71,4 \\
-71,4 \\
-60,5\end{array}$ & $\begin{array}{l}0,21 £ \\
0,21 £ \\
0,00 \dagger\end{array}$ \\
\hline $\begin{array}{l}\text { Cardiotocografia intermitente } \\
\text { Indução do TP }\end{array}$ & $\begin{array}{l}36,6 \\
12,2\end{array}$ & $\begin{array}{l}17,9 \\
6,2\end{array}$ & $\begin{array}{l}-51,1 \\
-48,4\end{array}$ & $\begin{array}{l}0,02 \dagger \\
0,35 £\end{array}$ \\
\hline Tonsura & 41,5 & 25,5 & $-38,6$ & $0,04 \dagger$ \\
\hline Bola obstétrica & 65,9 & 45,5 & $-31,0$ & $0,03 \dagger$ \\
\hline Escalda-pés & 14,6 & 13,1 & $-10,3$ & $1,00 \dagger$ \\
\hline $\begin{array}{l}\text { Aromaterapia } \\
\text { Posição semissentada }\end{array}$ & $\begin{array}{l}61,0 \\
63,4\end{array}$ & $\begin{array}{l}55,9 \\
60,0\end{array}$ & $\begin{array}{l}-8,4 \\
-5,4\end{array}$ & $\begin{array}{l}0,68 \dagger \\
0,00 £\end{array}$ \\
\hline Deambulação & 97,6 & 93,8 & $-3,9$ & $0,69 £$ \\
\hline
\end{tabular}

\section{Discussão}

Nesta seção serão discutidas as práticas assistenciais que apresentaram significância estatística, com aumento ou redução percentual, quando comparado os anos de 2013 e 2016.

Nesse estudo constatou-se aumento percentual de práticas assistenciais recomendadas pela OMS, ${ }^{(5)}$ como a utilização de partograma, com aumento de $43,3 \%$, a dieta líquida por via oral de $11,5 \%$, a mudança de posição de $828,6 \%$, rebozo de $167,3 \%$, as posiçôes de cócoras, de quatro apoios, lateral esquerda e direita de $100 \%$ e a massagem terapêutica de $33,4 \%$. Ressalta-se o aumento de $16,7 \%$ para a prática de amniotomia, cujo uso rotineiro não é recomendado pela OMS. ${ }^{(5)}$

O clampeamento tardio do cordão umbilical e o contato pele a pele (CPP), práticas benéficas para o recém-nascido. ${ }^{(6,7)}$ e recomendadas pela OMS, ${ }^{(5)}$ também apresentaram aumento percentual significativo (37,3\% e 33,2\%, respectivamente).

O partograma permite o monitoramento da evoluçáo do TP. Apresenta variação quanto à frequência da sua utilização nas maternidades brasileiras, atingindo taxas de $77,4 \%,{ }^{(8)} 48,3 \%{ }^{(9)}$ e $39,4 \% .{ }^{(10)}$ Destaca-se que ele pode contribuir para redução da duração do TP, de exames vaginais, da taxa de cesarianas e para melhora nos resultados maternos e neonatais, ${ }^{(11)}$ sendo assim, acredita-se que sua utilização deve ser estimulada.

A dieta líquida por via oral foi ofertada para a maior parte das parturientes deste estudo, constituindo um avanço na assistência ao parto, tendo em vista que as mulheres saudáveis têm um risco extremamente baixo de aspiração durante o parto, incluindo o parto cirúrgico, e levando em conta os benefícios da dieta no TP, como a reposição das necessidades energéticas, prevenção de cetose, hiponatremia e estresse materno. ${ }^{(12)}$ Nas maternidades brasileiras, a frequência de oferecimento de dieta por via oral foi inferior aos achados deste estudo, com taxas de $54,6 \%{ }^{(8)} 32,7 \%,{ }^{(13)} 26,7 \%{ }^{(9)}$ e $25,6 \%$. $^{(14)}$

A prática de mudança de posição no TP apresentou crescimento percentual expressivo e está relacionada a melhor evolução do TP e ao bem-estar materno e fetal, com diminuição da sensação dolorosa e aumento da satisfação materna, especialmente quando adotadas as posiçóes verticais e lateralizadas. ${ }^{(15,16)}$

As posiçóes verticais no período expulsivo foram predominantes nos partos assistidos pelas enfermeiras obstétricas, cujas posiçóes resultam em redução da duração do segundo estágio do TP, das taxas de episiotomia e de parto instrumental. No entanto, pode haver risco de perda sanguínea superior a $500 \mathrm{ml}$ e risco aumentado de laceraçóes perineais espontâneas de $2^{\circ}$ grau nas posiçôes verticais. ${ }^{(17)}$ Tradicionalmente as posiçóes de parto instituídas são as não verticais, pela prática profissional e por questóes socioculturais das mulheres, por acreditar que essa é a melhor ou a única possiblidade de posição do parto. Estudos apontam avanços na utilização de posiçóes verticais, ${ }^{(15,16)}$ o que reflete ser uma prática benéfica, com o aumento da sua utilização.

A massagem terapêutica no TP proporciona conforto, relaxamento e alívio da dor. Em materni- 
dades brasileiras a frequência de utilização da massagem foi de $34,8 \%{ }^{(16)}$ e $19,5 \%{ }^{(18)}$ sendo descrita como uma prática benéfica, de baixo custo, que pode reduzir o uso de medicamentos anestésicos e analgésicos. ${ }^{(18)}$

Outra tecnologia obstétrica não invasiva muito utilizada nesse estudo foi o rebozo, que consiste em uma técnica tradicional mexicana de massagem pélvica, para corrigir o posicionamento do feto ${ }^{(19)}$ além de ser eficaz na redução da dor e proporcionar uma experiência clínica e psicológica positiva para a mulher. ${ }^{(20)}$

Quanto à amniotomia, apesar do aumento percentual na taxa, essa prática não foi utilizada de forma rotineira nas parturientes (2,4\% em 2013 e $2,8 \%$ em 2016). A amniotomia pode estar associada a complicaçóes potenciais, como a ocorrência de desaceleraçóes da frequência cardíaca fetal, prolapso de cordão e infecção. ${ }^{(3)}$ Nas maternidades brasileiras a amniotomia apresenta taxas elevadas, de $67,1 \%,{ }^{(8)} 51,2 \%,{ }^{(13)} 40,7 \%,{ }^{(14)}$ e 27,3\%. ${ }^{(21)} \mathrm{A}$ análise sobre amniotomia obtida em revisão sistemática da Cochrane, evidenciou menor probabilidade da necessidade desta intervenção nas mulheres com parto assistido por enfermeiras obstétricas e obstetrizes, quando comparadas às atendidas em outros modelos de assistência. ${ }^{(22)}$ Esse achado é compatível com os indicadores encontrados no presente estudo, quando comparados às taxas de amniotomia encontradas na realidade brasileira, em partos assistidos no modelo biomédico. ${ }^{(13,14)}$ Esta revisão sugere ainda, menor probabilidade das mulheres atendidas em um modelo de assistência obstétrica liderado por enfermeiras obstétricas e obstetrizes de sofrerem intervençóes, além de maior probabilidade no nível de satisfação da mulher e uma probabilidade semelhante na ocorrência de desfechos adversos maternos ou perinatais.

Desta forma, seria esperada a redução do máximo possível de intervençóes nos partos assistidos por enfermeiras obstétricas, o que indica a necessidade de avanços no que tange à realização de amniotomia na instituição estudada, ainda que sejam baixos os percentuais e que não seja uma prática rotineira, quando comparada à realidade brasileira.

Em relação ao recém-nascido $(\mathrm{RN})$, constatouse que o clampeamento tardio do cordão umbilical foi realizado na maior parte da amostra. Tal prática permite a transfusáo placentária ao $\mathrm{RN}$, previne a anemia na infância e a hemorragia pós-parto. ${ }^{(5,6)}$ São escassas as publicaçóes com indicadores sobre essa prática. Em uma publicação brasileira a taxa de clampeamento tardio do cordão umbilical foi de $76 \%$. $^{(18)}$

O CPP imediatamente após o nascimento foi realizado em todos os $\mathrm{RN}$ estudados, sendo a maioria com duração de uma hora ou mais. Mulheres que realizaram CPP são mais propensas a amamentar exclusivamente da alta hospitalar até seis meses após o nascimento, têm maior probabilidade de aleitamento materno na primeira hora de vida, escores mais elevados de estabilidade do sistema cardiorrespiratório e maiores níveis de glicose no sangue para o RN. ${ }^{(7)}$ Nas maternidades brasileiras a taxa de CPP foi de $73,1 \%{ }^{(18)}$ e $43,3 \%$. $^{(13)}$

Algumas das práticas recomendadas pela $\mathrm{OMS}^{(5)}$ apresentaram redução percentual significativa, como o uso de medicamentos para alívio da dor e a analgesia epidural, ambas com reduçáo de 79,0\%.

A analgesia epidural não foi utilizada rotineiramente entre as parturientes. Nas maternidades brasileiras a analgesia epidural foi utilizada por $31,5 \%,^{(14)} 14 \%{ }^{(8)}$ e $9,1 \%{ }^{(13)}$ das parturientes. Ela aumenta a duração do período expulsivo e pode aumentar a taxa de cesariana e de parto instrumental. No entanto, não está associada a desfechos maternos ou perinatais adversos, motivo pelo qual o seu uso é justificado no TP. ${ }^{(23)}$ Em revisão sistemática, as mulheres atendidas no modelo liderado por enfermeiras obstétricas e obstetrizes tiveram menor probabilidade de receber analgesia regional, o que vai ao encontro dos achados do presente estudo. ${ }^{(22)}$ Houve diminuição percentual considerável dessa prática no comparativo dos anos, fato positivo, que pode estar relacionado ao aumento da utilização de métodos não invasivos, evitando-se o uso de intervenções com maior potencial de complicaçóes.

Poucas parturientes utilizaram medicamentos para alívio da dor, sendo os mais utilizados a Dipirona, a Hioscina e a Meperidina. O uso de Hioscina está relacionado ao alívio da dor no TP e à redução do primeiro estágio do TP, com aumento da dilatação cervical ${ }^{(24)}$ e a Meperidina está associada à redução da dor no TP, porém com ocorrência 
de náuseas, vômitos e sonolência materna e maior necessidade de uso de ocitocina. ${ }^{(25)}$ A prescrição de medicamentos para alívio da dor é realizada pelo médico obstetra, quando necessário, mediante avaliação da parturiente, quando as tecnologias obstétricas não invasivas não são suficientes para alívio da dor. Verificou-se uma redução importante no uso de medicamentos, o que permitiu às parturientes recorrer ao maior uso de tecnologias obstétricas não invasivas.

Entre as tecnologias obstétricas não invasivas, destaca-se o uso da bola para promover conforto e alívio da dor, favorecer a posição vertical e a progressão do TP. ${ }^{(18)}$ Em estudo brasileiro com partos assistidos por enfermeiras obstétricas, a bola foi utilizada por $54,6 \%$ das parturientes. ${ }^{(18)} \mathrm{Na}$ instituição em estudo, houve redução do uso da bola obstétrica no ano de 2016, possivelmente relacionado à redução do espaço físico, em decorrência de reforma da área física do centro obstétrico neste mesmo ano, o que limitou a realização dessa prática.

Entre as práticas não recomendadas pela OMS, ${ }^{(5)}$ houve redução percentual significativa da tricotomia $(-100 \%)$, supositório retal $(-85,8 \%)$, posição litotômica $(-85 \%)$, administração de ocitocina $(-73,3 \%)$, cateterização venosa $(-60,5 \%)$, cardiotocografia intermitente $(-51,1 \%)$, tonsura $(-38,6 \%)$ e posição semissentada $(-5,4 \%)$.

A tricotomia foi uma prática eliminada do rol de intervenções da instituição em estudo no ano de 2016, já a administração de supositório retal - utilizado para esvaziamento retal, porém sem evidências científicas de sua indicação no TP - e a tonsura utilizada para redução dos pelos pubianos, entretanto sem evidências científicas que justifiquem tal prática no $\mathrm{TP}$ - apresentaram redução percentual importante, sendo a última, devido ao seu caráter invasivo e ao risco de infecção. Acerca dessas práticas no TP não foram encontradas evidências científicas na literatura nacional e internacional, constituindo uma limitação do estudo.

A cardiotocografia (CTG) foi utilizada em uma pequena parcela de parturientes e de forma intermitente, o que permitiu a deambulação e mudança de posição no TP. Ensaio clínico randomizado mostrou que, quando comparado com a ausculta intermitente de batimentos cardiofetais, a CTG contínua não mostrou melhora significativa na taxa de mortalidade perinatal e houve aumento de cesarianas e partos vaginais instrumentais associados à CTG contínua. ${ }^{(26)}$

A cateterização venosa e a administração de ocitocina não foram utilizadas na maior parte das parturientes. A cateterização venosa não é recomendada por ser um procedimento invasivo que representa riscos para a mulher, além de prejudicar a sua mobilidade no TP. ${ }^{(5)}$ Nas maternidades brasileiras, a cateterização venosa foi utilizada em $73,8 \%{ }^{(15)} \mathrm{e}$ $54 \%{ }^{(10)}$ das parturientes e a administração de ocitocina em $52,2 \%,{ }^{(13)} 49,6 \%,{ }^{(21)} 41,7 \%,{ }^{(8)} 38,2 \%{ }^{(14)}$ e $27,6 \% .{ }^{(18)} \mathrm{O}$ uso de ocitocina, especialmente em doses elevadas e sem o monitoramento adequado, pode ocasionar sérios riscos para a mulher e o feto, como taquissistolia uterina e comprometimento da frequência cardíaca fetal, em decorrência do prolongamento da contratilidade uterina, que pode ocasionar diminuição do fluxo sanguíneo para o feto, estando associado à Índice de Apgar menor que sete no quinto minuto de vida, à hipotonia uterina e à hemorragia pós parto. ${ }^{(27)}$

Em relação à posição adotada pelas parturientes no período expulsivo, houve diminuição na posição litotômica e na posição semissentada, o que é positivo, uma vez que essas posiçóes devem ser desencorajadas, pois apresentam risco aumentado de edema vulvar e sangramento uterino acima de 500 $\mathrm{ml}$ após a dequitação placentária. ${ }^{(15)} \mathrm{Na}$ maior parte das maternidades brasileiras a posição litotômica é a predominante no período expulsivo, com frequências de $92 \%,{ }^{(14)} 77,1 \%{ }^{(28)}$ e de $66,8 \%,{ }^{(8)}$ e pode estar relacionada ao aumento de intervençóes durante o parto.

\section{Conclusão}

O presente estudo identificou altos índices de práticas assistenciais benéficas para as mulheres e para os recém-nascidos em partos assistidos por enfermeiras obstétricas. A comparação das práticas assistenciais nos anos estudados revelou redução de intervençóes como tricotomia, tonsura, supositório retal, posição 
litotômica, uso de medicamentos para alívio da dor, uso de ocitocina, analgesia epidural, cateterização venosa, cardiotocografia e posição semissentada; e aumento percentual de práticas como uso de partograma, mudança de posição, rebozo, posição de cócoras, posição de quatro apoios, posição lateral direita e esquerda, amniotomia, dieta líquida no TP, massagem terapêutica, clampeamento tardio do cordão umbilical e contato pele a pele. Ressalta-se que existem práticas assistenciais que carecem de avanços, como a redução da amniotomia, a qual, apesar de não ser realizada de maneira rotineira, foi uma intervenção com aumento percentual significativo no período estudado. Quanto à utilização da bola obstétrica foi constatado redução desta prática, contudo, ressalta-se que em condições habituais essa é uma das tecnologias obstétricas não invasivas mais utilizadas no trabalho de parto, sendo essa redução atribuída à reforma da área física do centro obstétrico durante o período estudado. A partir desses achados, o modelo colaborativo de assistência ao parto, com atuação das enfermeiras obstétricas, mostra-se como um caminho para a atenção às mulheres, com respeito à fisiologia do parto e ao protagonismo da mulher, capaz de promover redução de intervençôes desnecessárias, por meio da realização de práticas assistenciais que resultam em desfechos obstétricos e neonatais favoráveis.

\section{Colaborações}

Ritter SK, Gonçalves AC e Gouveia HG declaram que contribuíram com a concepção do estudo, análise e interpretação dos dados, redação do artigo, revisão crítica relevante do conteúdo intelectual e aprovação da versão final a ser publicada.

\section{Referências}

1. Vogt SE, Silva KS, Dias MA. Comparação de modelos de assistência ao parto em hospitais públicos. Rev Saude Publica. 2014;48(2):304-13.

2. Gama SG, Viellas EF, Torres JA, Bastos MH, Brüggemann OM, Theme Filha MM, et al. Labor and birth care by nurse with midwifery skills in Brazil. Reprod Health. 2016;13(Suppl 3):123.

3. Brasil. Ministério da Saúde. Diretriz Nacional de Assistência ao Parto Normal. Brasília (DF): Ministério da Saúde; 2016.
4. Downe S, Finlayson K, Fleming A. Creating a collaborative culture in maternity care. J Midwifery Womens Health. 2010;55(3):250-4.

5. World Health Organization (WHO). WHO recommendations: intrapartum care for a positive childbirth experience. Genebra: WHO; 2018.

6. Kc A, Rana N, Målqvist M, Jarawka Ranneberg L, Subedi K, Andersson 0 . Effects of delayed umbilical cord clamping vs early clamping on anemia in infants at 8 and 12 months: a randomized clinical trial. JAMA Pediatr. 2017;171(3):264-70.

7. Moore ER, Bergman N, Anderson GC, Medley N. Early skin-to-skin contact for mothers and their healthy newborn infants. Cochrane Database Syst Rev. 2016;11(6):CD003519.

8. Sousa AM, Souza KV, Rezende EM, Martins EF, Campos D, Lansky S. Práticas na assistência ao parto em maternidades com inserção de enfermeiras obstétricas, em Belo Horizonte, Minas Gerais. Esc Anna Nery. 2016;20(2):324-31.

9. Melo BM, Gomes LF, Henriques AC, Lima SK, Damasceno AK. Implementation of good practice in assistance to labor at a reference maternity. Rev Rene. 2017;18(3):376-82

10. Prado DS, Mendes RB, Gurgel RQ, Barreto ID, Bezerra FD, Cipolotti R, et al. Practices and obstetric interventions in women from a state in the Northeast of Brazil. Rev Assoc Med Bras (1992). 2017;63(12):1039-48.

11. Bedwell C, Levin K, Pett C, Lavender DT. A realist review of the partograph: when and how does it work for labour monitoring? BMC Pregnancy Childbirth. 2017;17(1):31.

12. Tillett J, Hill C. Eating and drinking in labor: reexamining the evidence. J Perinat Neonatal Nurs. 2016;30(2):85-7.

13. Monguilhott JJ, Brüggemann OM, Freitas PF, D'Orsi E. Nascer no Brasil: a presença do acompanhante favorece a aplicação das boas práticas na atenção ao parto na região Sul. Rev Saude Publica. 2018;52:1.

14. Leal MC, Pereira AP, Domingues RM, Theme Filha MM, Dias MA, Pereira MN, et al. Intervenções obstétricas durante 0 trabalho de parto e parto em mulheres brasileiras de risco habitual. Cad Saude Publica. 2014;30(1 Suppl 1):S17-32.

15. Schettini NJC, Griboski RA, Faustino AM. Partos normais assistidos por enfermeiras obstétricas: posição materna e a relação com lacerações perineais espontâneas. Rev Enferm UFPE on line. 2017;11(2):932-40.

16. Vargens OM, Silva AC, Progianti JM. Contribuição de enfermeiras obstétricas para consolidação do parto humanizado em maternidades no Rio de Janeiro-Brasil. Esc Anna Nery. 2017;21(1): e20170015.

17. Gupta JK, Sood A, Hofmeyr GJ, Vogel JP. Position in the second stage of labour for women without epidural anaesthesia. Cochrane Database Syst Rev. 2017;5(5):CD002006.

18. Medeiros RM, Teixeira RC, Nicolini AB, Alvares AS, Corrêa AC, Martins DP. Cuidados humanizados: a inserção de enfermeiras obstétricas em um hospital de ensino. Rev Bras Enferm. 2016;69(6):1091-8.

19. Cohen SR, Thomas CR. Rebozo Technique for Fetal Malposition in Labor. J Midwifery Womens Health. 2015;60(4):445-51.

20. Iversen ML, Midtgaard J, Ekelin M, Hegaard HK. Danish women's experiences of the rebozo technique during labour: A qualitative explorative study. Sex Reprod Healthc. 2017;11(1):79-85.

21. Reis CS, Souza D0, Nogueira MF, Progianti JM, Vargens OM. Análise de partos acompanhados por enfermeiras obstétricas na perspectiva da humanização do parto e nascimento. Rev Cuid Fundam Online. 2016;8(4):4972-9.

22. Sandall J, Soltani H, Gates S, Shennan A, Devane D. Midwife-led continuity models versus other models of care for childbearing women [Review]. Cochrane Database Syst Rev. 2016 Apr;4(4):CD004667. 
23. Piedrahíta-Gutiérrez DL, España-Chamorro JA, Piedrahíta-Gutiérrez WE, López-Clavijo CA, Henao-Flórez RE. Resultados obstétricos y perinatales en pacientes con 0 sin analgesia obstétrica durante el trabajo de parto. latreia. 2016;29(3):263-9.

24. Kirim S, Asicioglu 0, Yenigul N, Aydogan B, Bahat N, Bayrak M. Effect of intravenous hyoscine-N-butyl bromide on active phase of labor progress: a randomized double blind placebo controlled trial. J Matern Fetal Neonatal Med. 2015;28(9):1038-42.

25. Smith LA, Burns E, Cuthbert A. Parenteral opioids for maternal pain management in labour. Cochrane Database Syst Rev. 2018 Jun;6(5):CD007396.
26. Alfirevic Z, Devane D, Gyte GM, Cuthbert A. Continuous cardiotocography (CTG) as a form of electronic fetal monitoring (EFM) for fetal assessment during labour. Cochrane Database Syst Rev. 2017 Feb;2(1):CD006066.

27. Saraiva JP, Vogt SE, Rocha JS, Duarte ED, Simão DA. Associação entre fatores maternos e neonatais e o Apgar em recém nascidos de risco habitual. Rev Rene. 2018;19:e3179

28. Santos RC, Riesco ML. Implementação de práticas assistenciais para prevenção e reparo do trauma perineal no parto. Rev Gaucha Enferm. 2017; 6;37(Spe):e68304. 